\title{
A Simplified Model for Flagellar Motion
}

\author{
Kelsey Meinerz, John Karkheck \\ Department of Physics, Marquette University, Milwaukee ${ }^{1}$
}

\begin{abstract}
.
Many bacteria use long, thin appendages called Flagella to propel them forward. Examples of bacteria which use these are Escherichia coli and Salmonella typhimurium. A rotary motor within the cell causes the flagellum to spin, which then propels the bacterium through its fluid environment. This is a complex process, especially for a single-celled organism. In order to better understand how this process works, we have created and tested a model for flagellar motion using springs in different fluids. Quantifying the propulsive force is an important goal, not only for relating it to bacterial motion, but also for potential applications in low gravity environments.
\end{abstract}

\section{Background.}

A majority of the published research relating to flagella focus on how the flagella are self-assembled, how the motor within the cell causes the flagellum to rotate, and how the bacteria utilize their flagella to swim towards food or otherwise change direction through the process of "tumbling." There is very little research out there which reduces the process to a simpler model which can then be used to test different conditions and better understand how bacteria move. In his book Biological Physics: Energy, Information, Life (copyright 2004), Philip Nelson sought to explain how flagella work by comparing it to a helix twirling through the fluid in which the bacteria live. He used this idea to then explain how the net force from the flagellum on the fluid is reciprocated by the fluid which drives the bacterium forward. The fluid needs to be viscous enough to have a low Reynolds number so that the bacterium is not governed entirely by inertial forces. This topic was covered in the class titled The Physical Basis of Biological Structure and Function in the fall semester of 2011. Nelson's development focuses on a small segment of the helix to explain the existence of a propulsive force.
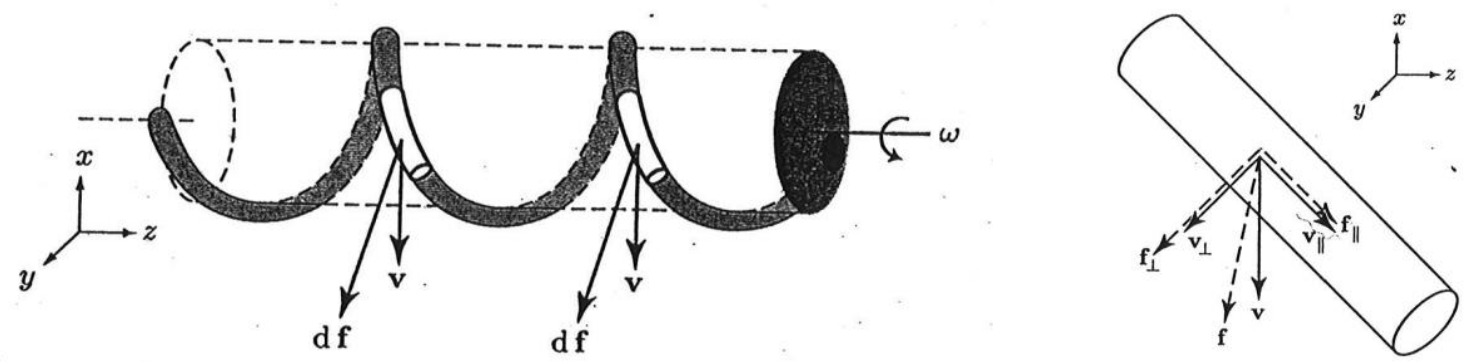

Figures 1 (left) \& 2 (right) courtesy of Philip Nelson's Biological Physics: Energy, Information, Life Updated First Edition; pages 175, 177; Figure 1 is an illustration of a rotating helix with a small segment highlighted; Figure 2 is that segment of the helix, illustrates the components of the force and velocity for that segment.

\footnotetext{
${ }^{1}$ Special thanks to the Wisconsin Space Grant Consortium for financial support. Special thanks as well to Joseph Holbus and Thomas Silman for their help in engineering this project.
} 
This project sought to create a simple, macroscopic model for a bacterial flagellum. This was to be done by testing the characteristics of a rigid, helix-shaped body being rotated about its axis in a fluid environment and comparing that to the known characteristics of a bacterial flagellum to see if the rigid body accurately models a flagellum. Not only would this be a way to better understand bacterial flagella, but this also could potentially be applied to make a gentle mixing system in a low-gravity environment.

\section{Method and Results.}

The first apparatus, shown in Figure 3, utilizes a twopulley system to drive the spring. One of the pulleys is a PASCO rotary motion sensor which measures the velocity and acceleration of the hanging bucket. The falling mass applies a torque to the second pulley which is mounted on a rotating shaft. The spring is mounted on the other end of the shaft, so when the pulley begins to rotate the spring does as well. The distinct springs were immersed in fluids of different viscosities. This set up allowed us to determine the tangential component of the force upon the spring through an analysis of the torques in the system. Data was collected using the program DataStudio, which is the software intended for PASCO instruments. The rotary motion sensor recorded the linear velocity of the system, from which it was possible to determine the linear acceleration of the system.

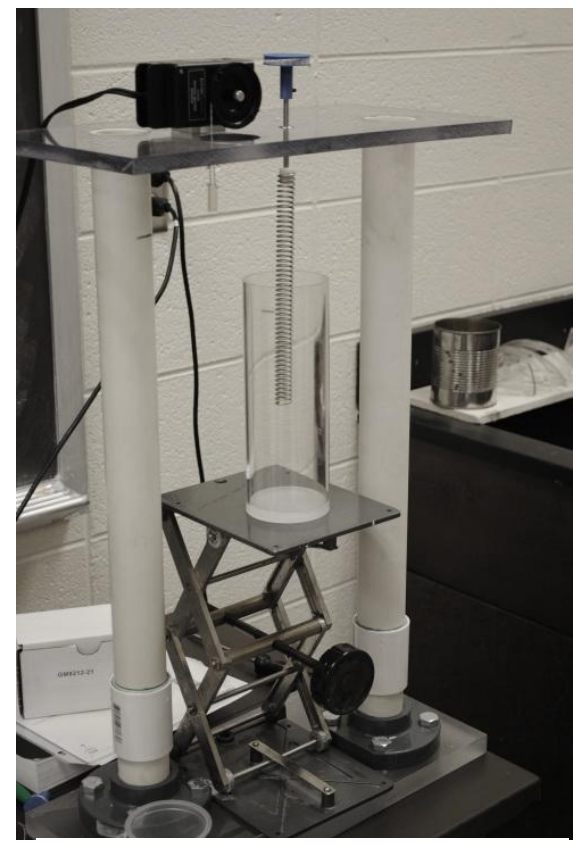

Figure 3: The first apparatus using the 1.50in pulley and the 8in spring

Changing viscosities. The first test which was performed using this apparatus involved immersing the springs in different fluids to see how the viscosity affected the motion of the spring. The first fluid used was air, then distilled $\mathrm{H} 2 \mathrm{O}$, and finally motor oil. Graphs of the hanging mass versus the acceleration were plotted for each of these fluids. The graphs show that as the viscosity of the fluid increased, the data became more systematic. This is because in a more viscous fluid, the spring did not whip as much and the rotation of the spring was more stable.
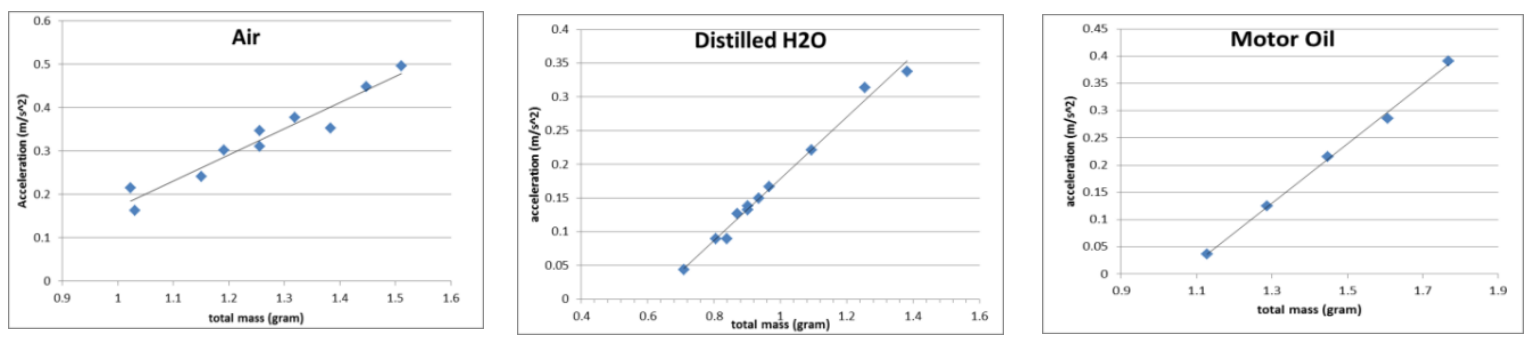

Figures 4 (left), 5 (center), \& 6 (right); Plots of the hanging mass versus the acceleration; Figure 4 is the graph for the spring rotating in air; Figure 5 is the graph for the spring rotating in distilled water; Figure 6 is the graph for the spring rotating in motor oil 
Changing pulley diameters. In an attempt to better understand the system, the diameter of the second pulley was changed and the effects of that change were studied. The diameters used were $0.75 \mathrm{in}, 1.50 \mathrm{in}$, and $2.00 \mathrm{in}$. Since the pulley with the smallest diameter has a smaller moment arm, it allowed the system to accelerate quickly. Typically, this resulted in the spring whipping and the data was not as systematic. The pulleys with larger diameters had larger moment arms, which resulted in slower accelerations. This resulted in more stable rotations of the springs and cleaner data.

Accelerating system. Graphs were made by plotting the acceleration achieved with a particular hanging mass. These were made for different wet lengths of the spring. Wet length refers to the length of spring which was immersed in the fluid. Using these graphs it was possible to extrapolate back to zero acceleration and determine what mass would, theoretically, cause the system to move without accelerating. A graph was then made illustrating the relationship between the wet length and the zero acceleration mass. Examples of these are shown in Figures 7 and 8. Using the equation for the line from the zero acceleration mass versus wet length graph, it was possible to gain information about the force on the spring.

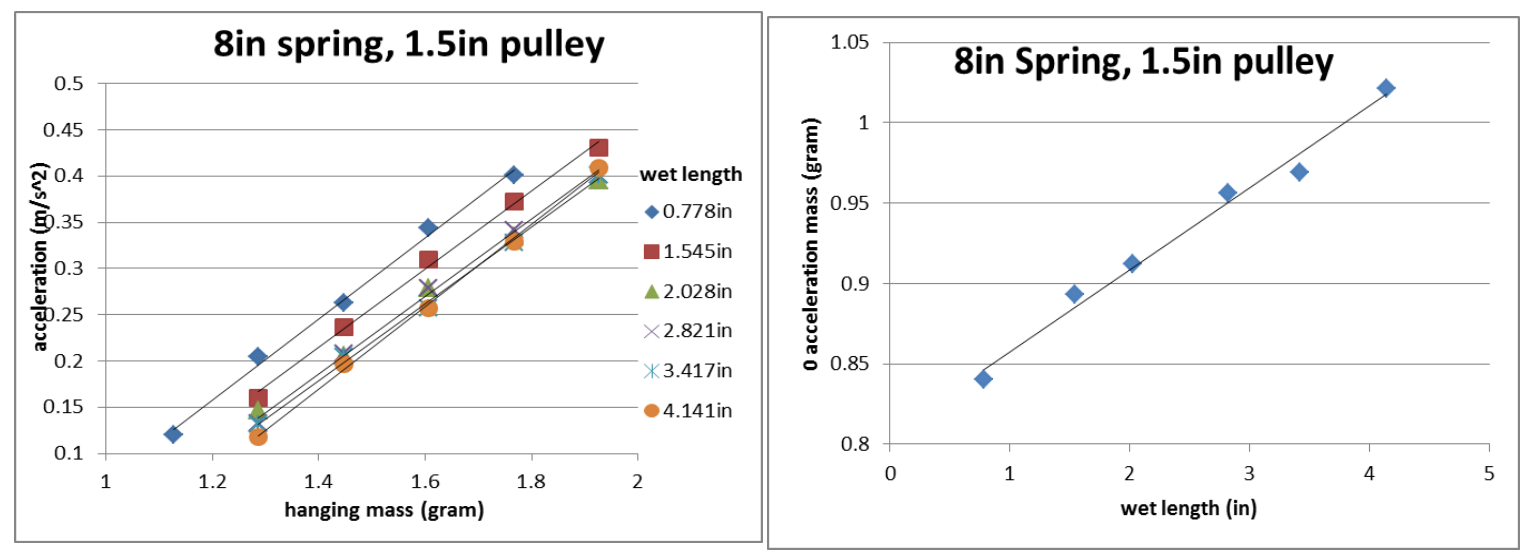

Figures 7 (left) \& 8 (right); Figure 7 is a plot of the hanging mass versus the acceleration, each line represents a different wet length; Figure 8 is a plot of the wet length versus the mass necessary to achieve zero acceleration

$$
\boldsymbol{f}=\frac{\boldsymbol{R} * \boldsymbol{g}}{\boldsymbol{X}} * \frac{\Delta \boldsymbol{m}}{\Delta \boldsymbol{l}}
$$

This is the equation for the force on the spring per unit length, where $\mathrm{R}$ is the radius of the pulley, $\mathrm{g}$ is gravity, $\mathrm{X}$ is the distance from the axis of rotation to the center of the spring, $\Delta \mathrm{m} / \Delta \mathrm{l}$ is the slope from the graph of 0 acceleration mass vs. wet length (Figure 8 ). 
System in free-fall. When running tests of the springs in the motor oil, many of the trials resulted in the hanging mass reaching a terminal velocity, meaning the system was no longer accelerating. Since this system is not accelerating, the drag force upon the spring is directly proportional to the force created by the hanging mass. Therefore, the torques created by each are equal and opposite. Figures 9 shows a trial in DataStudio which shows the plateau in the velocity versus time graph; showing that the system did achieve terminal velocity. Figure 10 is a graph of the terminal speed versus the amount of hanging mass for different wet lengths. The graph at right shows that the relationship between the hanging mass and the terminal speed is linear, which translates to a linear relationship between the drag force and the terminal speed.
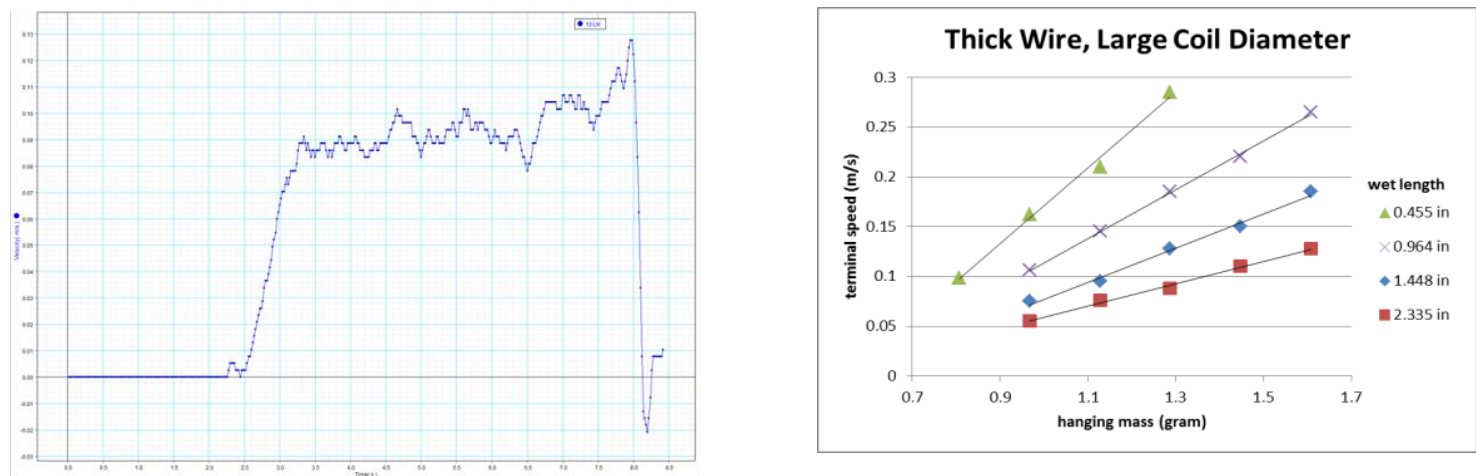

Figures 9 (left) \& 10 (right); Figure 9 is an example of the velocity versus time graph when the system reached an effectively constant speed; Figure 10 is a plot of the hanging mass versus the terminal speed for different wet lengths

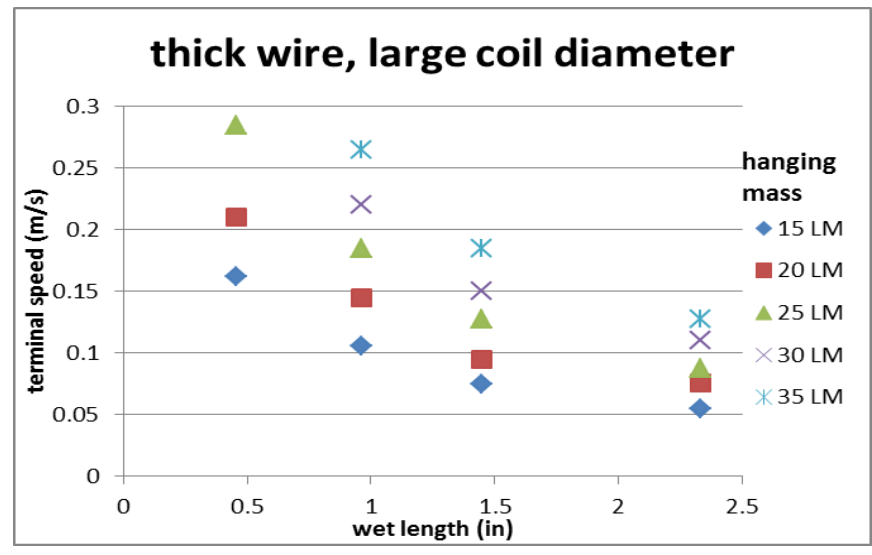

Figure 11; a plot of the wet length versus the terminal speed for different hanging masses
We also graphed the relationship between the terminal velocity and the wet length for different amounts of hanging masses. LM stands for large mass which were steel balls that were 32 milligrams and placed into a bucket which weighed 487 milligrams. The graph shows that as the wet length increases, the terminal speed decreases. 


\section{Conclusions.}

Based on the relationship between the viscosity and the stability of the rotation, it is likely that flagellar motion is stabilized in a viscous environment.

A typical result for the force per unit wet length of spring is $5.4 \mathrm{mN} / \mathrm{meter}$ in a water environment using the smaller vessel. Preliminary results using the larger vessel yield forces larger by a factor of ten. This suggests that boundary conditions have an effect upon the spring in the smaller vessel.

Based on the linear relationship between the hanging mass and the terminal speed in the non-accelerating system it was determined that the system is operating in the regime of Stokes flow.

Based on the relationship between the wet length and the terminal speed in the nonacceleration system, it is likely that there is a constraint on flagella length.

\section{Future Work.}

Figure 12 is the second apparatus for this project. This set up utilizes a DC motor with a speed control device. This will apply a constant torque to the shaft the spring is mounted on and will cause the spring to turn at a constant rate. This should create a vortex pattern which causes the surface of the liquid to act like a parabolic mirror. Using LASER pointers, the curvature of the mirror can be determined. This will allow us to ascertain the longitudinal force on the spring. This portion of the experiment is still a work in progress.

Data collected with the first apparatus using different spring geometries- pitch, coil diameter, and wire diameter- is under analysis.

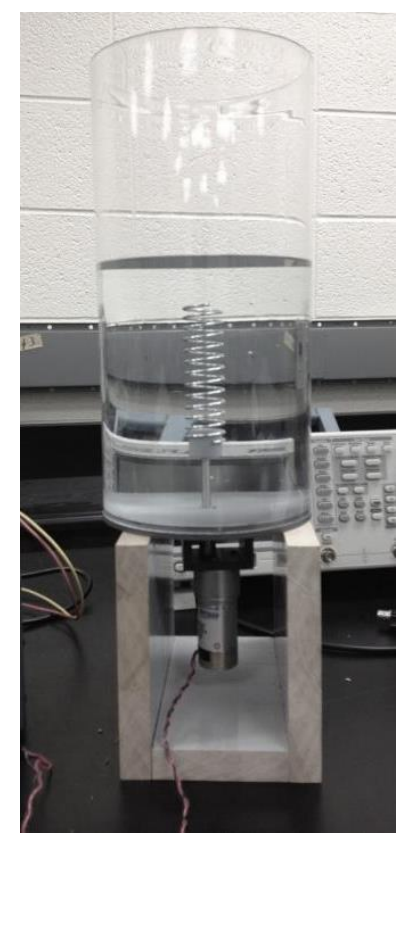

\section{References.}

Nelson, Philip. Biological Physics: Energy, Information, Life. New York, NY: W. H. Freeman and, 2004. Print

Figure 12: The second apparatus using a spring with a thicker coil diameter 